\title{
STABILITY ANALYSIS AND INTERNAL HEATING EFFECT ON OSCILLATORY CONVECTION IN A VISCOELASTIC FLUID SATURATED POROUS MEDIUM UNDER GRAVITY MODULATION
}

\author{
B.S. BHADAURIA*, M.K. SINGH, A. SINGH and B.K. SINGH \\ Department of Applied Mathematics, School of Physical Sciences \\ Babasaheb Bhimrao Ambedkar University, Lucknow-226025, INDIA \\ E-mails:mathsbsb@yahoo.com; s.manojbbau@gmail.com \\ ajaysingh0044@gmail.com; bksingh0584@gmail.com \\ P. KIRAN \\ Department of Applied Mathematics, Rayalaseema University \\ Kurnool, Andhra Pradesh-518007, INDIA \\ E-mail: kiran40p@gmail.com
}

\begin{abstract}
In this paper, we investigate the combined effect of internal heating and time periodic gravity modulation in a viscoelastic fluid saturated porous medium by reducing the problem into a complex non-autonomous GinzgburgLandau equation. Weak nonlinear stability analysis has been performed by using power series expansion in terms of the amplitude of gravity modulation, which is assumed to be small. The Nusselt number is obtained in terms of the amplitude for oscillatory mode of convection. The influence of viscoelastic parameters on heat transfer has been discussed. Gravity modulation is found to have a destabilizing effect at low frequencies and a stabilizing effect at high frequencies. Finally, it is found that overstability advances the onset of convection, more with internal heating. The conditions for which the complex Ginzgburg-Landau equation undergoes Hopf bifurcation and the amplitude equation undergoes supercritical pitchfork bifurcation are studied.
\end{abstract}

Key words: Non-linear stability analysis, complex Ginzburg-Landau equation, gravity modulation, internal heating, bifurcation.

\section{Introduction}

The studies of thermal instability in porous media play a very significant role in many areas such as in petroleum industry, chemical engineering and geophysics, etc. A detailed account of the work on thermal instability in porous media has been given in the most excellent books due to Ingham and Pop [1], Nield and Bejan [2] and Vafai [3].

External regulation of convection is important either for enhancing or diminishing heat transfer in a physical system. This type of regulation (e.g., thermal, gravity, rotation and magnetic field modulation) is used effectively to control convective phenomenon. This paper is concerned with the thermal instability under gravity modulation, first studied by Gresho and Sani [4]. In this model they observed that the gravity modulation enables the system to get control on its instability either by suitably adjusting the values of frequency or the amplitude of modulation. The related studies of gravity modulation are given in [5]-[16]. The nature of non-Newtonian fluids is quite different from Newtonian fluids due to their properties such as like shear stress and shear strain. The basic idea for considering these fluids is due to their oscillatory nature. For example, industrial fluids are basically non-Newtonian. In particular, viscoelastic fluids have been important. A proper understanding of convective motion and its behaviour is necessary for controlling many

\footnotetext{
* To whom correspondence should be addressed
} 
processes such as geothermal reservoirs, filtration, and enhanced oil recovery. Green [17] was the first to study the oscillatory convection in a viscoelastic fluid layer, Vest and Arpaci [18] reported the occurrence of overstability for typical Rayleigh-Benard convection of a horizontal homogeneous Maxwellian fluid layer heated from below.

Bhatia and Steiner [19] studied thermal instability in a rotating viscoelastic fluid layer. Some studies on thermal convection of viscoelastic fluids in porous media are available in the literature. Kim et al. [20] investigated the critical conditions of stationary and oscillatory instabilities in a horizontal porous layer saturated with a viscoelastic fluid based on the modified Darcy-Oldroyd model. Malashetty and Kulkarni [21] used a two-field model in the energy equation to investigate the thermal non-equilibrium problem of a Maxwell fluid in a porous medium, Wang and Tan [22] performed linear and nonlinear analyses on the double-diffusive convection with the Soret effect in a Maxwell fluid-saturated porous medium.

Kumar and Bhadauria [23]-[25] studied thermal instability in a rotating porous layer saturated with a viscoelastic fluid and with double diffusive gradients. An oscillatory mode of convection is investigated by Bhadauria and Kiran [26]-[29]. These authors derived complex Ginzburg-Landau equations for obtaining finite amplitude convection under modulation, and the conditions for which the oscillatory convective flow is confirmed.

There are situations of great practical importance where the porous material offers its own source of heat. This gives a different way in which a convective flow can be set up through the local heat generation within the porous media. Such a situation can occur through radioactive decay or through, in the present perspective, a relatively weak exothermic reaction which can take place within the porous material.

To the best of our knowledge, there is no work related to the oscillatory convection under modulation with an internal heat source in the literature. It is of great practical importance, when the porous material offers its own source of heat and the internal heating can modify the convective flow through local heat generation within the porous media. To be specific, internal heat is one of the main sources of energy for celestial bodies caused by nuclear fusion and decaying of radioactive materials which keep the celestial objects warm and active. It is due to the internal heating of the earth that there exists a thermal gradient between the interior and exterior of the earth crust, saturated by multi-components fluids, which helps convective flow, thereby transferring the thermal energy towards the surface of the earth. So the role of internal heat generation becomes very important in several applications that include geophysics, reactor safety analyses, fire and combustion studies, and in storage of radioactive materials. Some of the studies on thermal instability under internal heat generation are due to [30-40]. The present study aims at finding the combined effect of gravity modulation and internal heating on oscillatory convection in a porous material saturated with a viscoelastic fluid.

\section{Mathematical formulation}

An infinitely extended horizontal porous medium saturated with a viscoelastic fluid heated from below and cooled from above is considered. The Darcy model has been employed in the momentum equation. Further, an internal heat source term has been included in the energy equation. The physical configuration of the model is reported in Fig.1. The corresponding mathematical model of the problem under the Boussinesq approximation [26] is as follows

$$
\begin{aligned}
& \nabla \cdot \boldsymbol{q}=0, \\
& \left(\bar{\lambda}_{1} \frac{\partial}{\partial t}+1\right)(-\nabla p+\rho \boldsymbol{g})-\frac{\mu}{K}\left(\bar{\lambda}_{2} \frac{\partial}{\partial t}+1\right) \boldsymbol{q}=0, \\
& \frac{\partial T}{\partial t}+(\boldsymbol{q} \cdot \nabla) \mathrm{T}=\kappa_{T} \nabla^{2} T+Q\left(T-T_{0}\right), \\
& \rho=\rho_{0}\left\{1-\alpha_{T}\left(T-T_{0}\right)\right\}
\end{aligned}
$$


where the physical variables have their usual meanings as given in the nomenclature. The externally imposed gravitational field and the thermal boundary conditions are given by

$$
\begin{gathered}
\boldsymbol{g}=g_{0}\left\{1+\chi^{2} \delta \cos (\Omega t)\right\} \hat{k}, \\
T=\left\{\begin{array}{cc}
T_{0}+\Delta T, & \text { at } \quad z=0, \\
T_{0}, & \text { at } \quad z=d
\end{array}\right.
\end{gathered}
$$

where $g_{0}$ is the mean gravity and $\hat{k}$ is the unit vector along the positive $z$ axis.

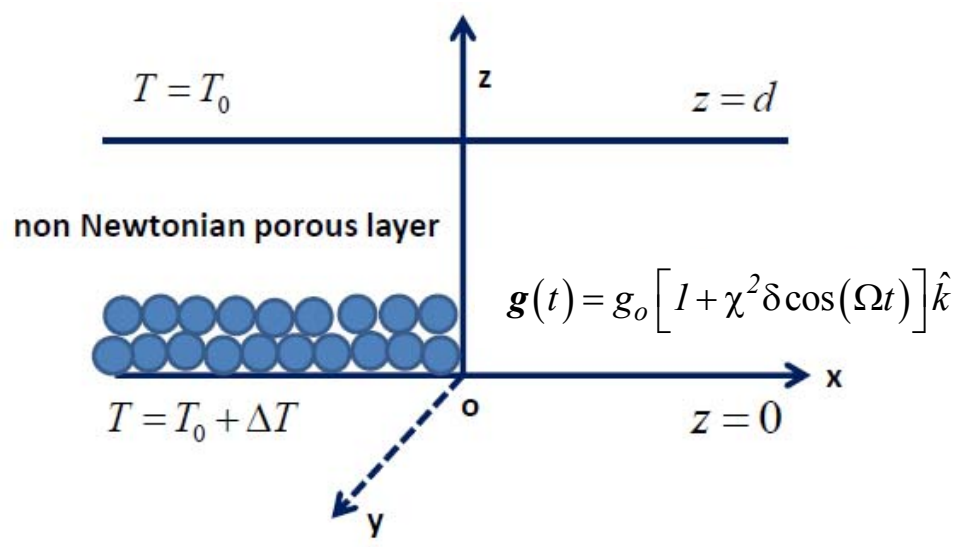

Fig.1. Physical configuration of the problem.

\section{Basic state}

At this state the velocity, pressure, temperature and density profiles are given by

$$
\boldsymbol{q}_{b}=0, \quad p=p_{b}(z), \quad T=T_{b}(z), \quad \rho=\rho_{b}(z) .
$$

The following relations follow is direct from Eq.(3.1) and Eq. (2.1)

$$
\begin{aligned}
& \frac{\partial p_{b}}{\partial z}=-\rho_{b} \boldsymbol{g}, \\
& \kappa_{T} \frac{d^{2}\left(T_{b}-T_{0}\right)}{d z^{2}}+Q\left(T_{b}-T_{0}\right)=0, \\
& \rho_{b}=\rho_{0}\left\{1-\alpha_{T}\left(T_{b}-T_{0}\right)\right\} .
\end{aligned}
$$

The solution of Eq.(3.3), subject to the boundary conditions (2.3), is given by 


$$
T_{b}=T_{0}+\Delta T \frac{\sin \left(\left(\sqrt{\frac{Q}{\kappa_{T}}}\right)\left(1-\frac{z}{d}\right)\right)}{\sin \left(\sqrt{\frac{Q}{\kappa_{T}}}\right)} .
$$

Superimpose the finite amplitude perturbations on the basic state in the form

$$
\boldsymbol{q}=q_{b}+q^{\prime}, \quad T=T_{b}+T^{\prime}, \quad p=p_{b}+p^{\prime}, \quad \rho=\rho_{b}+\rho^{\prime}
$$

where the primes represent the perturbation quantities. The dimensionless governing system as in [26] is given by

$$
\begin{aligned}
& \left(\lambda_{1} \frac{\partial}{\partial t}+1\right) g_{m} R_{a D} \frac{\partial T}{\partial x}+\left(\lambda_{2} \frac{\partial}{\partial t}+1\right) \nabla^{2} \psi=0, \\
& -\frac{\partial \psi}{\partial x} \frac{\partial T_{b}}{\partial z}+\left(\frac{\partial}{\partial t}-\nabla^{2}-R_{i}\right) T=\frac{\partial(\psi, T)}{\partial(x, z)}
\end{aligned}
$$

where $g_{m}=\left(1+\chi^{2} \delta \cos (\Omega t)\right), R_{a D}=\frac{\alpha_{T} g_{0} \Delta T K d}{\nu \kappa_{T}}$ is the thermal Rayleigh number, $R_{i}=\frac{Q d^{2}}{\kappa_{T}}$ is the internal Rayleigh number, $v=\frac{\mu}{\rho_{0}}$ is the kinematic viscosity. The above system will be solved by considering stress free and isothermal boundary conditions as given below

$$
\psi=\frac{\partial^{2} \psi}{\partial z^{2}}=T=0 \quad \text { on } \quad z=0, \quad z=1 .
$$

The dimensionless basic temperature field $T_{b}(z)$ from Eq.(3.8) is given by

$$
\frac{d T_{b}}{d z}=-\frac{\sqrt{R_{i}} \cos \left(\sqrt{R_{i}}(1-\mathrm{z})\right)}{\sin \left(\sqrt{R_{i}}\right)}
$$

Introducing a small perturbation parameter $\chi$, a deviation from the critical state of onset of convection, the variables for a weak non-linear state is expanded in the power series in $\chi$ as $[44,45]$

$$
\begin{aligned}
& R_{a D}=R_{0}+\chi^{2} R_{2}+\chi^{4} R_{4}+\cdots, \\
& \psi=\chi \psi_{1}+\chi^{2} \psi_{2}+\chi^{3} \psi_{3}+\ldots . \\
& T=\chi T_{1}+\chi^{2} T_{2}+\chi^{3} T_{3}+\ldots
\end{aligned}
$$


where $R_{0}$ is the critical value of the Darcy-Rayleigh number at which the onset of convection takes place in the absence of gravity modulation.

\section{Analysis of the periodic solutions}

In order to study time periodic convective phenomenon according to [20,26], the slow and fast time scale will be introduced by $\frac{\partial}{\partial t}=\frac{\partial}{\partial \tau}+\chi^{2} \frac{\partial}{\partial s}$. The above system (3.7) and (3.8) will be solved at every order of $\chi$. case)

At the first order, we obtain the matrix operator (the reader may note that this is similar to the linear

$$
\left[\begin{array}{cc}
\left(\lambda_{2} \frac{\partial}{\partial \tau}+1\right) \nabla^{2} & R_{0}\left(\lambda_{1} \frac{\partial}{\partial \tau}+1\right) \frac{\partial}{\partial x} \\
-\frac{\partial}{\partial x} \frac{\partial T_{b}}{\partial z} & \left(\frac{\partial}{\partial \tau}-\nabla^{2}-R_{i}\right)
\end{array}\right]\left[\begin{array}{l}
\psi_{1} \\
T_{1}
\end{array}\right]=\left[\begin{array}{l}
0 \\
0
\end{array}\right]
$$

The solution of the first order system with the boundary conditions (3.9) is assumed as:

$$
\begin{aligned}
& \psi_{1}=\left(A(s) e^{i \omega \tau}+\bar{A}(s) e^{-i \omega \tau}\right) \sin a x \sin \pi z, \\
& T_{1}=\left(B(s) e^{i \omega \tau}+\bar{B}(s) e^{-i \omega \tau}\right) \cos a x \sin \pi z
\end{aligned}
$$
expression

The undetermined amplitudes are functions of slow time scale, and are related by the following

$$
B(s)=-\frac{4 \pi^{2} a}{\left(c+i \omega-R_{i}\right)\left(4 \pi^{2}-R_{i}\right)} A(s)
$$

where

$$
c=a^{2}+\pi^{2}
$$

The values of the critical Darcy-Rayleigh number and the corresponding wave number of the system for a stationary mode of convection are as given below

$$
\begin{aligned}
& R_{0}^{s t}=\frac{c\left(\mathrm{c}-R_{i}\right)\left(4 \pi^{2}-R_{i}\right)}{4 \pi^{2} a^{2}}, \\
& a_{c}^{2}=\sqrt{\pi^{4}-\pi^{2} R_{i}} .
\end{aligned}
$$

For the system without internal-heating, that is, $R_{i}=0$, we get

$$
R_{0}=\frac{c^{2}}{a^{2}}
$$




$$
a_{c}=\pi
$$

which are same as the classical results obtained by Chandrasekhar [41]. Moreover, the critical DarcyRayleigh number and associated wave number of the system for the oscillatory mode of convection are obtained as

$$
\begin{aligned}
& R_{0}^{o s c}=\left(\frac{c\left(c-R_{i}\right)-\lambda_{2} \omega^{2}}{a^{2}}\right) \frac{4 \pi^{2}-R_{i}}{4 \pi^{2}}, \\
& a_{c}^{2}=\sqrt{\pi^{4}-\pi^{2} R_{i}+\frac{\pi^{2}}{\lambda_{2}}}
\end{aligned}
$$

where $\omega$ is the oscillatory frequency, given as

$$
\omega^{2}=\frac{\left(\lambda_{1}-\lambda_{2}\right) c+R_{i}\left(\lambda_{2}-\lambda_{1}\right)-1}{\lambda_{1} \lambda_{2}} .
$$

Since $\omega$ is real, and so, from relation (4.11), the necessary condition for oscillatory convection is obtained as

$$
\lambda_{1}>\lambda_{2}+\frac{1-R_{i}\left(\lambda_{2}-\lambda_{1}\right)}{c}
$$

Now, at the second order, we have

$$
\left[\begin{array}{cc}
\left(\lambda_{2} \frac{\partial}{\partial \tau}+1\right) \nabla^{2} & R_{0}\left(\lambda_{1} \frac{\partial}{\partial \tau}+1\right) \frac{\partial}{\partial x} \\
-\frac{\partial}{\partial x} \frac{\partial T_{b}}{\partial z} & \left(\frac{\partial}{\partial \tau}-\nabla^{2}-R_{i}\right)
\end{array}\right]\left[\begin{array}{l}
\psi_{2} \\
T_{2}
\end{array}\right]=\left[\begin{array}{l}
R_{21} \\
R_{22}
\end{array}\right]
$$

where

$$
\begin{aligned}
& R_{21}=0 \\
& R_{22}=\frac{\partial \psi_{1}}{\partial x} \frac{\partial T_{1}}{\partial z}-\frac{\partial \psi_{1}}{\partial z} \frac{\partial T_{1}}{\partial x}
\end{aligned}
$$

The second order solution, subject to the boundary condition (3.9), is given by

$$
\begin{aligned}
& \psi_{2}=0 \\
& \left(\frac{\partial}{\partial \tau}-\nabla^{2}-R_{i}\right) T_{2}=R_{22} .
\end{aligned}
$$

Keeping $[20,26]$ in mind, the second order temperature term written as 


$$
T_{2}=\left\{T_{20}+T_{22} \mathrm{e}^{2 i \omega \tau}+\bar{T}_{22} \mathrm{e}^{-2 i \omega \tau}\right\} \sin (2 \pi \mathrm{z})
$$

where $T_{22}$ and $T_{20}$ are temperature fields having the terms with the frequency $2 \omega$ and independent of fast time scale, respectively. The solutions of the second order problems are

$$
\begin{aligned}
& T_{20}=\frac{\pi a}{8 \pi^{2}-2 R_{i}}\{A(s) \bar{B}(s)+\bar{A}(s) B(s)\}, \\
& T_{22}=\frac{\pi a}{8 \pi^{2}+4 i \omega-2 R_{i}} A(s) B(s) .
\end{aligned}
$$

The horizontally averaged Nusselt number, $\mathrm{Nu}(s)$, for the oscillatory mode of convection is given by

$$
\mathrm{Nu}(s)=1+\left[\chi^{2}\left(\frac{\partial T_{2}}{\partial z}\right)_{z=0} /\left(\frac{\partial T_{b}}{\partial z}\right)_{z=0}\right]
$$

By using Eqs (3.10), (4.18)-(4.20), we can simplify Eq.(4.21) as

$$
\begin{aligned}
& \mathrm{Nu}(s)=1+\left[\frac{4\left(c-R_{i}\right) \pi^{2} a^{2}}{\left(\left(c-R_{i}\right)^{2}+\omega^{2}\right)\left(8 \pi^{2}-2 R_{i}\right)}+\right. \\
& \left.\frac{2 \pi^{2} a^{2}}{\sqrt{\left(c-R_{i}\right)^{2}+\omega^{2}} \sqrt{\left(8 \pi^{2}-2 R_{i}\right)^{2}+16 \omega^{2}}}\right]\left(\frac{4 \pi^{2}}{4 \pi^{2}-R_{i}}\right) \frac{\tan \sqrt{R_{i}}}{\sqrt{R_{i}}}|A(s)|^{2} .
\end{aligned}
$$

It is clear that gravity modulation is effective at third order and affects $\mathrm{Nu}(s)$ through $A(s)$ which is evaluated at third order.

At the third order, we have

$$
\left[\begin{array}{cc}
\left(\lambda_{2} \frac{\partial}{\partial \tau}+1\right) \nabla^{2} & \mathrm{R}_{0}\left(\lambda_{1} \frac{\partial}{\partial \tau}+1\right) \frac{\partial}{\partial x} \\
-\frac{\partial}{\partial x} \frac{\partial T_{b}}{\partial z} & \left(\frac{\partial}{\partial \tau}-\nabla^{2}-R_{i}\right)
\end{array}\right]\left[\begin{array}{l}
\psi_{3} \\
T_{3}
\end{array}\right]=\left[\begin{array}{c}
R_{31} \\
R_{32}
\end{array}\right]
$$

where

$$
\begin{aligned}
& R_{31}=-\lambda_{2} \frac{\partial}{\partial s}\left(\nabla^{2} \psi_{1}\right)-R_{0} \lambda_{1} \frac{\partial}{\partial s} \frac{\partial T_{1}}{\partial x}-\left(R_{2}+R_{0} \delta \cos (\Omega \mathrm{s})\right)\left(\lambda_{1} \frac{\partial}{\partial \tau}+1\right) \frac{\partial T_{1}}{\partial x}, \\
& R_{32}=\frac{\partial \psi_{1}}{\partial x} \frac{\partial T_{2}}{\partial z}-\frac{\partial T_{1}}{\partial s} .
\end{aligned}
$$

Using first and second order solutions, the expressions of $R_{31}$ and $R_{32}$ will be determined. Now, using the solvability condition for the existence of third order solution, we derive the complex GingburgLandau equation for finite amplitude convection. 


$$
\frac{d A(s)}{d s}-\gamma^{-1} F(s) A(s)+\gamma^{-1} k|A(s)|^{2} A(s)=0
$$

where

$$
\begin{aligned}
& \gamma=\lambda_{2} c-\frac{4 R_{2} \lambda_{1} a^{2} \pi^{2}}{\left(c+i \omega-R_{i}\right)\left(4 \pi^{2}-R_{i}\right)}+\frac{4 R_{2} a^{2} \pi^{2}\left(1+i \omega \lambda_{1}\right)}{\left(c+i \omega-R_{i}\right)^{2}\left(4 \pi^{2}-R_{i}\right)}, \\
& F(s)=\frac{4 R_{2} a^{2} \pi^{2}\left(1+i \omega \lambda_{1}\right)(1+\delta \cos (\Omega \mathrm{s}))}{\left(c+i \omega-R_{i}\right)\left(4 \pi^{2}-R_{i}\right)}
\end{aligned}
$$

and

$$
\begin{aligned}
& k=-\left(\frac{4 \pi^{2} a^{4} R_{2}\left(1+\lambda_{1} i \omega\right)\left(c-R_{i}\right)}{\left(c+i \omega-R_{i}\right)\left(\left(c-R_{i}\right)^{2}+\omega^{2}\right)\left(8 \pi^{2}-2 R_{i}\right)}+\right. \\
& \left.+\frac{4 \pi^{2} a^{4} R_{2}\left(\left(c-R_{i}\right)^{2}+\omega^{2} \lambda_{1}\right)}{\left(c+i \omega-R_{i}\right)\left(\left(c-R_{i}\right)^{2}+\omega^{2}\right)\left(8 \pi^{2}-2 R_{i}+4 i \omega\right)}\right) \frac{4 \pi^{2}}{4 \pi^{2}-R_{i}} .
\end{aligned}
$$

Writing $A(s)$ in the phase-amplitude form, we get

$$
A(s)=|A(s)| e^{i \varphi}
$$

Now, substituting Eq.(4.27) in Eq.(4.26), we get the following expression for the amplitude $|A(s)|$ as

$$
\begin{aligned}
& \frac{d|A(\mathrm{~s})|^{2}}{d s}-2 p_{r}|A(s)|^{2}+2 l_{r}|A(s)|^{4}=0, \\
& \frac{d(\operatorname{ph}(\mathrm{A}(s)))}{d s}=p_{i}-l_{i}|A(s)|^{2}
\end{aligned}
$$

where $\gamma^{-1} F(\mathrm{~s})=p_{r}+i p_{i}, \gamma^{-1} k=l_{r}+i l_{i} \quad$ and $\mathrm{ph}($.$) represents the phase shift. Equation (4.28) solved$ numerically using the function NDSolve of Mathematica, subject to the suitable initial condition $A(0)=a_{0}$, where $a_{0}$ is the chosen initial amplitude of convection. In our computation, we assume $R_{2}=R_{0}$ to keep the parameters to a minimum.

\section{Bifurcation analysis}

A qualitative study of a dynamical system is very important, thus it has attracted attention of researchers during the last few decades. Bifurcations are important scientifically, since they which provide models of transitions and instabilities when as some control parameter is varied [42]. In the present paper, two types of bifurcation (1) Pitchfork bifurcation (this bifurcation is common in physical problems that have 
a symmetry); (2) Hopf bifurcation (bifurcation corresponding to the presence of $\lambda_{1,2}= \pm i \omega_{0}, \omega_{0}>0$ ) are discussed, for details cf. [43].

\subsection{Hopf bifurcation}

The complex Ginzburg-Landau Eq.(4.26) can be written as

$$
\frac{d A(s)}{d s}=(\alpha+i \beta) A(s)-(\xi+i \varsigma)|A(s)|^{2} A(s)=0
$$

where

$$
\alpha=\operatorname{Re}\left[\frac{F(s)}{\gamma}\right], \quad \beta=\mathrm{I}_{m}\left[\frac{F(s)}{\gamma}\right], \quad \xi=\operatorname{Re}\left[\frac{k}{\gamma}\right], \quad \text { and } \quad \varsigma=I_{m}\left[\frac{k}{\gamma}\right]
$$

Putting $A(s)=\rho_{l} e^{i \phi}$ in Eq.(5.1) and equating the real and imaginary parts, we get

$$
\begin{aligned}
& \dot{\rho}_{l}=\rho_{l}\left(\alpha-\xi \rho_{l}^{2}\right), \\
& \dot{\phi}=\beta-\varsigma \rho_{l}^{2} .
\end{aligned}
$$

Clearly, Eq.(5.2) has an equilibrium point $\rho_{l}=0$, for all values of $\alpha$. Further, Eq.(5.3) describes the rotation. Hence, Eq.(5.1) has an equilibrium at the point $A(s)=0$, i.e., origin. This equilibrium is a stable focus for $\alpha<0$ and an unstable focus for $\alpha>0$ and at $\alpha=0$ (critical value) the equilibrium is non-linearly stable and topologically equivalent to the focus. This equilibrium is surrounded, for $\alpha>0$, by an isolated limit cycle that is unique and stable. All orbits starting outside or inside the cycle except at the origin tend to the cycle as $s \rightarrow+\infty$, see Fig.2. This is an Andronov-Hopf bifurcation. Here, we assume $\lambda_{1}$ as the bifurcation parameter, the critical value of the Hopf-bifurcation is $\lambda_{1}=0.14336936$. The phase portrait diagram for the critical value shows that the system (5.1) undergoes Hopf-bifurcation around the origin (see Fig.3). This bifurcation can also be represented in the $(x, y, \alpha)$ coordinate system see Fig.4. Figure 5 shows that the $\alpha$ family of limit cycles forms a paraboloid surface.

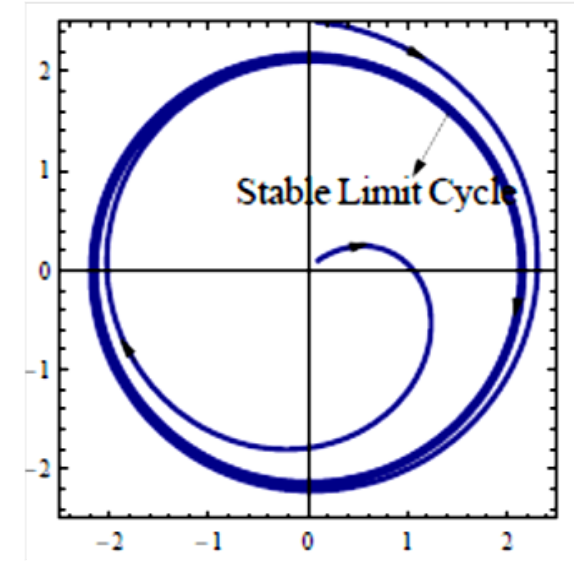

Fig.2. Stable limit cycle for $\lambda_{1}$ greater than critical value. $\lambda_{1}=0.5, \lambda_{2}=0.1, \Omega=1, \delta=0.02, R_{i}=0.4$. 


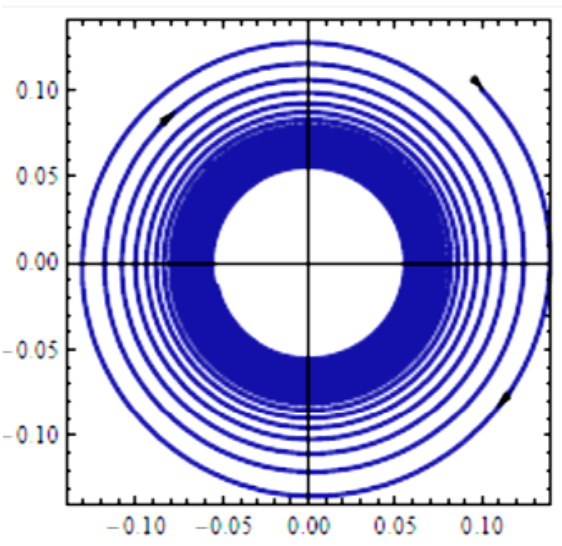

Fig.3. Hopf bifurcation w.r.t. bifurcation parameter $\lambda_{1} \cdot \lambda_{1}=0.14336936, \lambda_{2}=0.1, \Omega=1, \delta=0.02, R_{i}=0.4$.

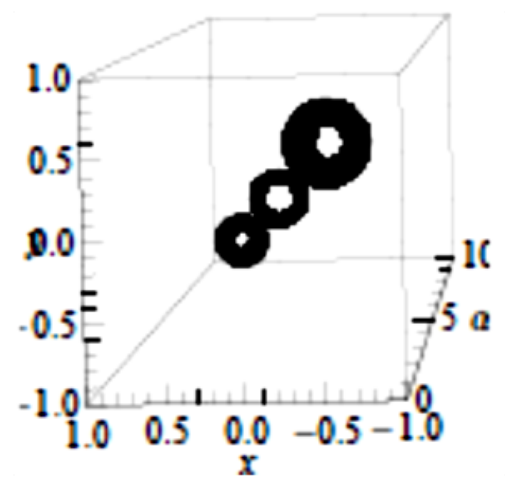

Fig.4. Supercritical Hopf bifurcation in the phase parameter $(x, y, \alpha)$ space.

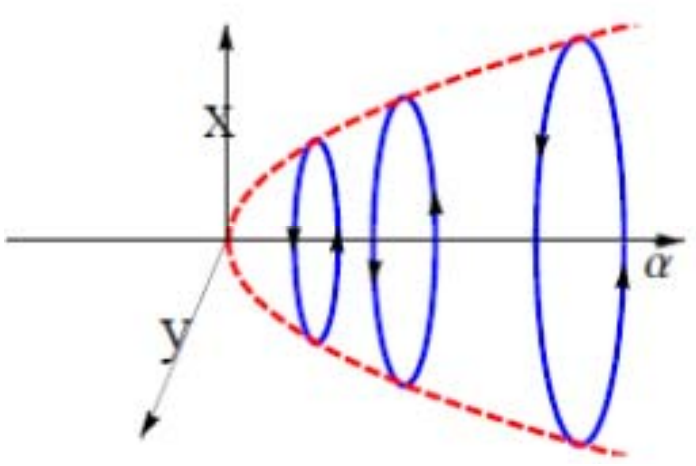

Fig.5. Paraboloid surface formed by $\alpha$ family of limit cycles shown in Fig.4. 


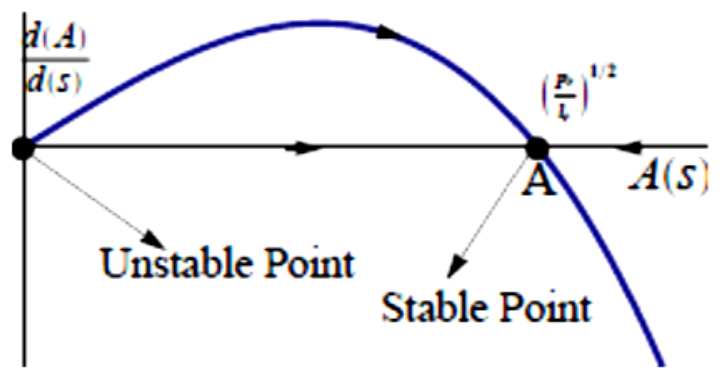

Fig.6. The point $A$ is stable while the origin is unstable. $\lambda_{1}=0.5, \lambda_{2}=0.1, \Omega=2, \delta=0.02, R_{i}=1$.

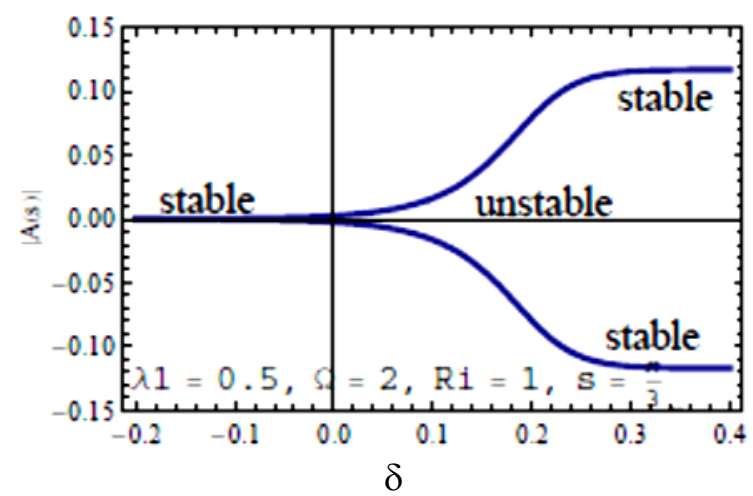

Fig.7. Supercritical Pichfork Bifurcation. $\lambda_{1}=0.5, \lambda_{2}=0.1, \Omega=2, \delta=0.02, R_{i}=1, s=\frac{\pi}{3}$.

\subsection{Pitchfork bifurcation}

Eq.(4.28) can be written as

$$
\frac{d|A(s)|}{d s}-p_{r}|A(s)|+l_{r}|A(s)|^{3}=0
$$

The steady state solution (equilibrium point) of Eq.(5.4) is $|A(s)|=0$ for all values of $p_{r}, l_{r}$ and $|A(s)|= \pm \sqrt{\frac{p_{r}}{l_{r}}}$ for $p_{r}>0, l_{r}>0$.

The unsteady state solution of Eq.(5.4) is given by

$$
|A(s)|^{2}=\frac{A_{0}^{2}}{\frac{l_{r}}{p_{r}} A_{0}^{2}+\left(1-\frac{l_{r}}{p_{r}} A_{0}^{2}\right) e^{-2 p_{r} s}}, \quad p_{r}>0, \quad l_{r}>0
$$

where $A_{0}$ is the initial value of the amplitude. From Eq.(5.5), it is clear that as $s \rightarrow-\infty,|A(s)| \rightarrow 0$ and if $s \rightarrow \infty$, then $|A(s)|$ grows towards $\sqrt{\frac{p_{r}}{l_{r}}}$ whenever $0<A_{0}<\sqrt{\frac{p_{r}}{l_{r}}}$ and decreases towards $\sqrt{\frac{p_{r}}{l_{r}}}$ whenever 
$A_{0}>\sqrt{\frac{p_{r}}{l_{r}}}$. Thus, the equilibrium point $A(s)=0$ is the only equilibrium point whenever $p_{r}<0$ and it is stable. If $\operatorname{Pr}=0$, then the origin of the only equilibrium point is still stable. When $\operatorname{Pr}>0$ and $l_{r}>0$ then $|A(s)|=0$ is still an equilibrium point, but becomes unstable and two new stable equilibrium points appear on either side of $|A(s)|=0$, symmetrically located at $|A(s)|= \pm \sqrt{\frac{p_{r}}{l_{r}}}$, Fig.14. This is known as supercritical pitchfork bifurcation, depicted in Fig.7.

\section{Results and discussion}

This paper investigates the combined effect of internal heating and gravity modulation on oscillatory convection in a porous medium saturated with a viscoelastic fluid. A weakly nonlinear stability analysis is performed to investigate the effect of gravity modulation on heat transport in the presence of internal heat generation. This analysis helps us to derive an amplitude equation in terms of a complex coefficient which gives a finite amplitude of convection. The present model is valid for oscillatory mode of convection whenever the relation (28) holds for the viscoelastic parameters. The new contribution to the recent study by Bhadauria and Kiran [26] is the effect of heat generation and bifurcation analysis. The results of the corresponding problem are depicted in Figs 8-13, where the graphs are drawn for $\mathrm{Nu}$ versus slow time $s$. Relation Eq.(4.22) confirms that the value of $\mathrm{Nu}$ starts with 1, thus showing the conduction state initially. The value of $\mathrm{Nu}$ increases as time varies, thus showing the convection state; becomes oscillatory, thus showing the modulation effect. The numerical values of $\mathrm{Nu}$ have been obtained from the expression (4.22) while solving the amplitude Eq.(4.28).

The effect of the internal Rayleigh number $R_{i}$ on heat transport is to increase the heat transport in the system as depicted in Fig.8. It is to be noted that when the value of $R_{i}$ increases, the internal energy of the system increases. The moderate values of $R_{i}$ are considered to avoid domination in the system. This confirms the results obtained recently in the studies $[34,35]$. The effects of relaxation parameter $\lambda_{1}$ (see, Fig.9) and retardation parameter $\lambda_{2}$ (see, Fig.10) are found to destabilize or stabilize the system, respectively, and consequently increase or decrease the heat transfer in the system. The effects of the amplitude $\delta$ and frequency $\Omega$ of modulation on heat transport are given in Fig.11 and Fig.12, respectively. Figure 11 confirms that an increment in the amplitude of modulation increases the magnitude of $\mathrm{Nu}$, and so, enhances the heat transfer and advances the onset of convection. An opposite effect is obtained in the case of frequency of modulation, see Fig.12. Moreover, the effect of gravity modulation decreases as the frequency of modulation increases. The effect disappears altogether when the frequency of modulation becomes very large. The proposed results of internal heating have been compared with the results of noninternal heating system, reported in Fig.13. It is observed that in the presence of an internal heat source, the magnitude of $\mathrm{Nu}$ is larger than that in the absence of internal-heating, i.e., the heat transport in the system is due to internal-heating. Thus, internal-heating advances the onset of convection, confirming the results obtained by Bhadauria et al. [36]-[38]. 


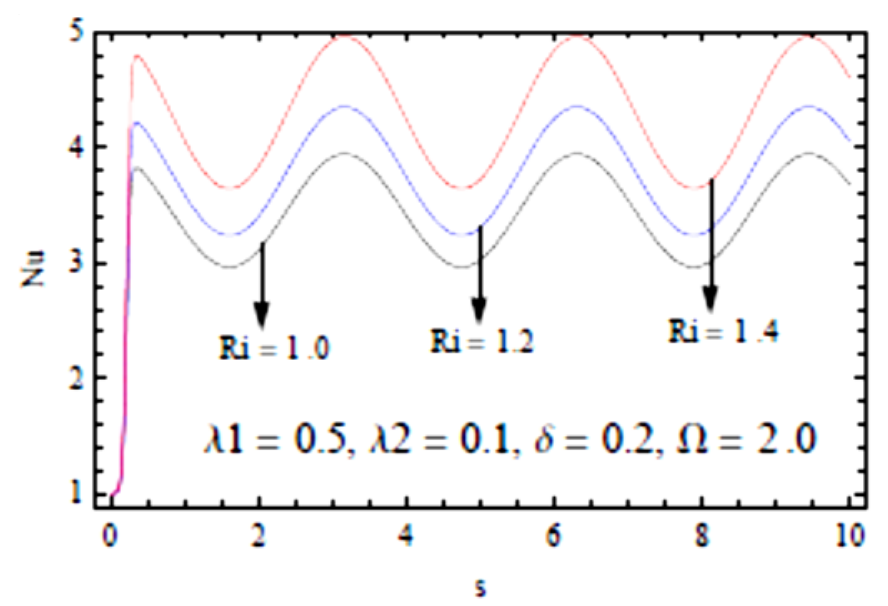

Fig.8. Effect of $R_{i}$ on $\mathrm{Nu}$ for fixed values of the other parameters.

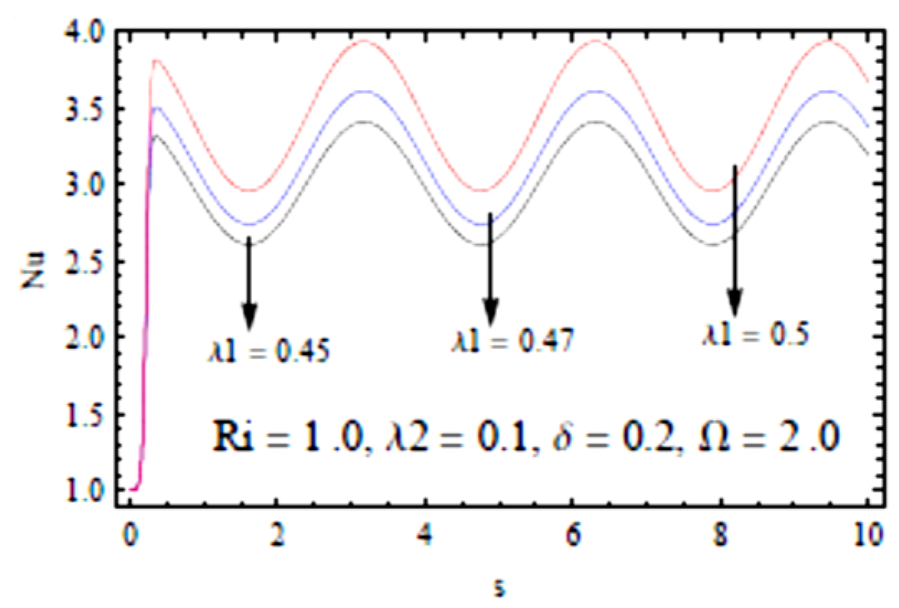

Fig.9. Effect of $\lambda_{l}$ on $\mathrm{Nu}$ for fixed values of the other parameters.

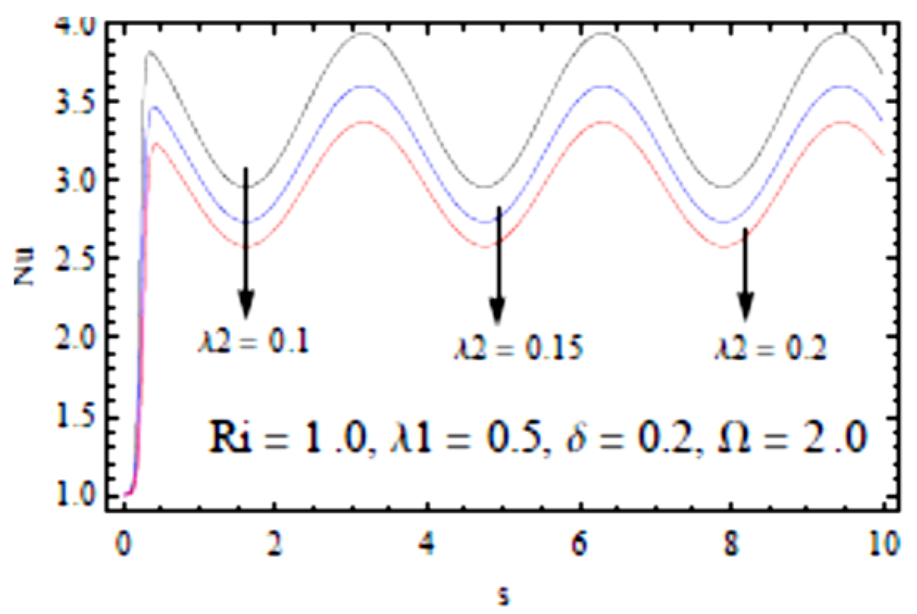

Fig.10. Effect of $\lambda_{2}$ on $\mathrm{Nu}$ for fixed values of the other parameters. 


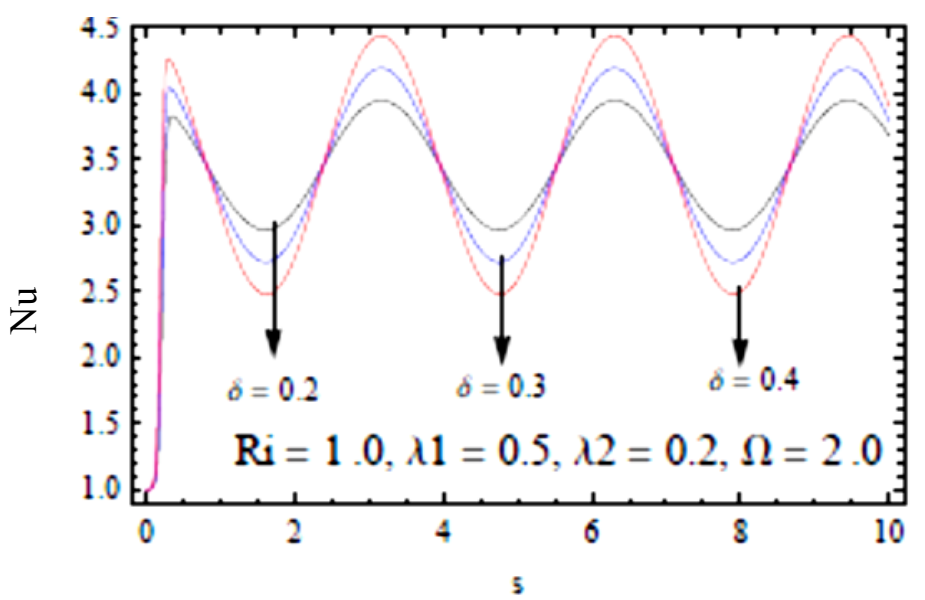

Fig.11. Effect of $\delta$ on $\mathrm{Nu}$ for fixed values of the other parameters.

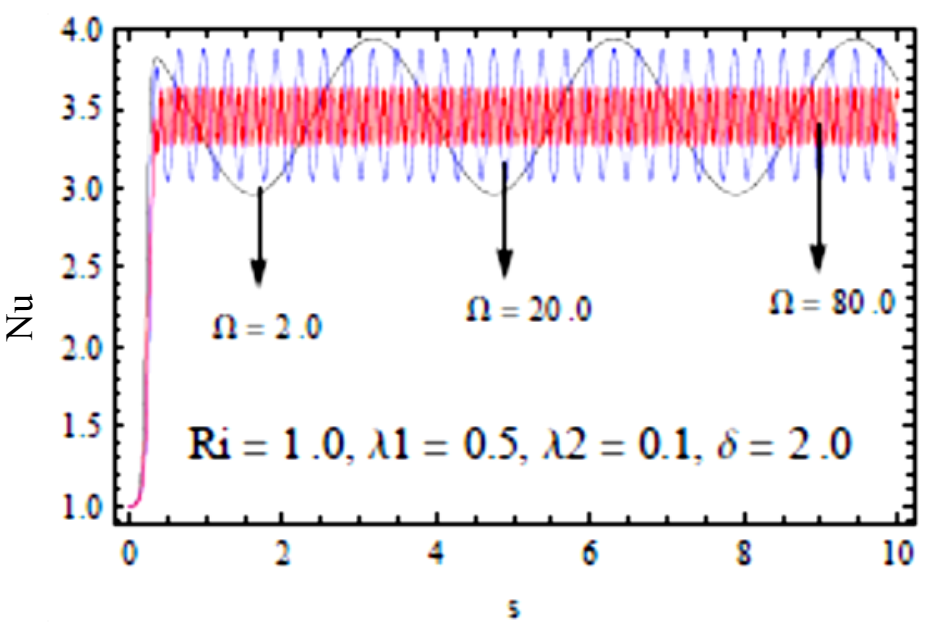

Fig.12. Effect of $\Omega$ on $\mathrm{Nu}$ for fixed values of the other parameters.

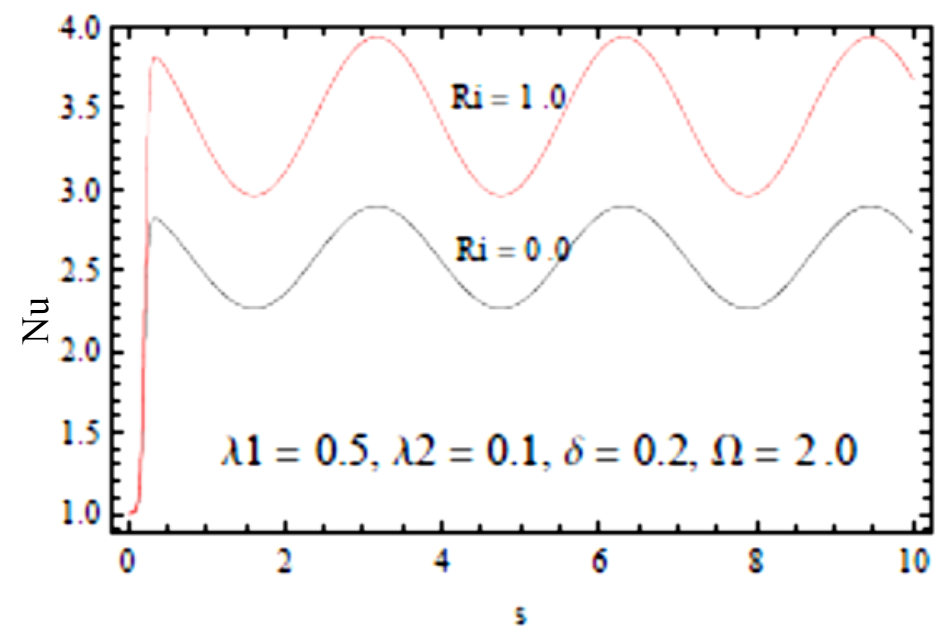

Fig.13. Comparison between internal and non internal-heating system. 
In Figs 14-15, the streamlines and corresponding isotherms are depicted, respectively, at $s=0.0,0.13,0.16,0.2,0.3,0.43$ for $\lambda_{1}=0.5, \lambda_{2}=0.1, \delta=0.2, \Omega=2, \chi=0.5$, and $R_{i}=0.1$. From these figures, it is observed that initially when time is small, the magnitude of streamlines is also small [Figs $14 \mathrm{a}, \mathrm{b}]$ and isotherms are straight [Figs $15 \mathrm{a}, \mathrm{b}]$, that is the system is in conduction state. However, as time increases, the magnitude of streamlines increases [Figs 14c, d] and the isotherms lose their evenness [Figs $15 \mathrm{c}, \mathrm{d}]$. This shows that convection is taking place in the system. The system achieves the steady state beyond $s=0.2$ as there is no change in the streamlines and isotherms, [Figs $14 \mathrm{e}, \mathrm{f}$ and $15 \mathrm{e}, \mathrm{f}$ ].
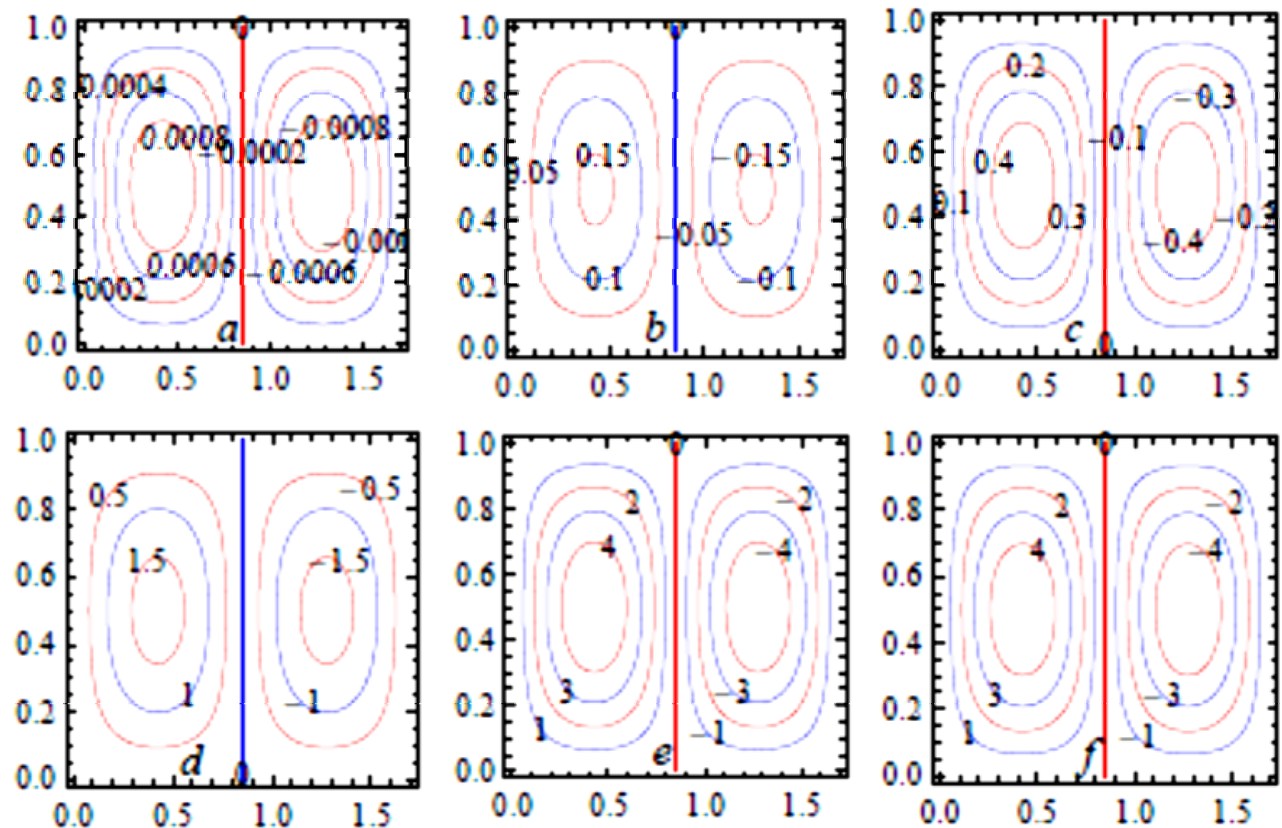

Fig.14. Streamlines at (a) $s=0.0$,

(b) $s=0.13,(\mathrm{c}) \mathrm{s}=0.16,(\mathrm{~d}) s$

(d) $s=0.2$, (e) $s=0.3$, (f) $s=0.43$.
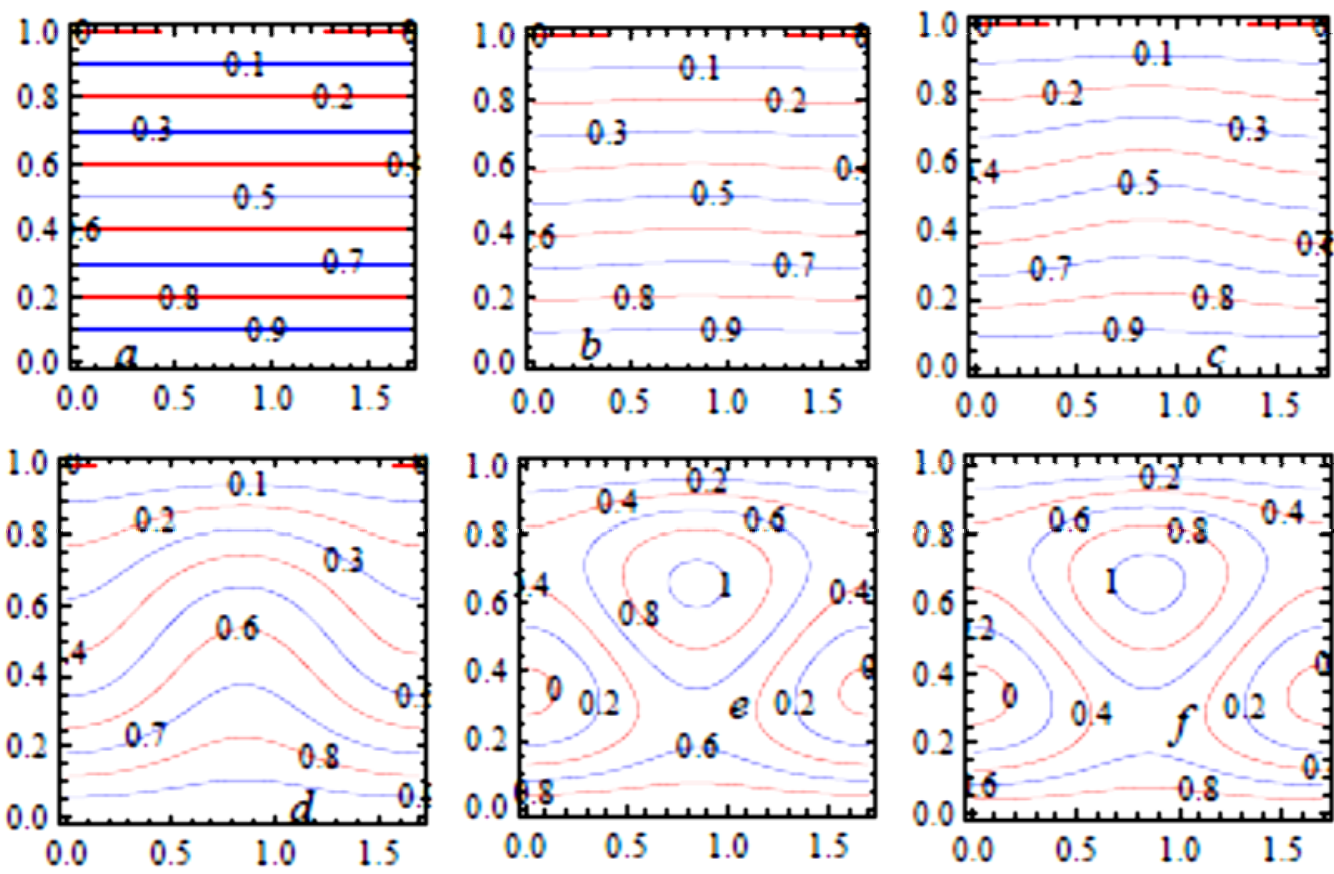

Fig.15. Isotherms at (a) $s=0.0$, (b) $s=0.13$, (c) $s=0.16$, (d) $s=0.2$, (e) $s=0.3$, (f) $s=0.43$. 
Also, by taking $\lambda_{1}$ as the bifurcation parameter, we have shown that the system represented by Landau Eq.(4.26) undergoes Hopf bifurcation. Further, the limit cycles are found to be stable circles. A sketch of this bifurcation in the $(x, y, \alpha)$ coordinate plane, results in a paraboloid surface. Apart from the Hopf bifurcation, we have also shown that the system represented by the amplitude Eq.(4.28) undergoes the supercritical pichfork bifurcation. Figure 16 shows that as $s$ increases the amplitude $|A(s)|$ becomes stable and remains stable for all future of time.

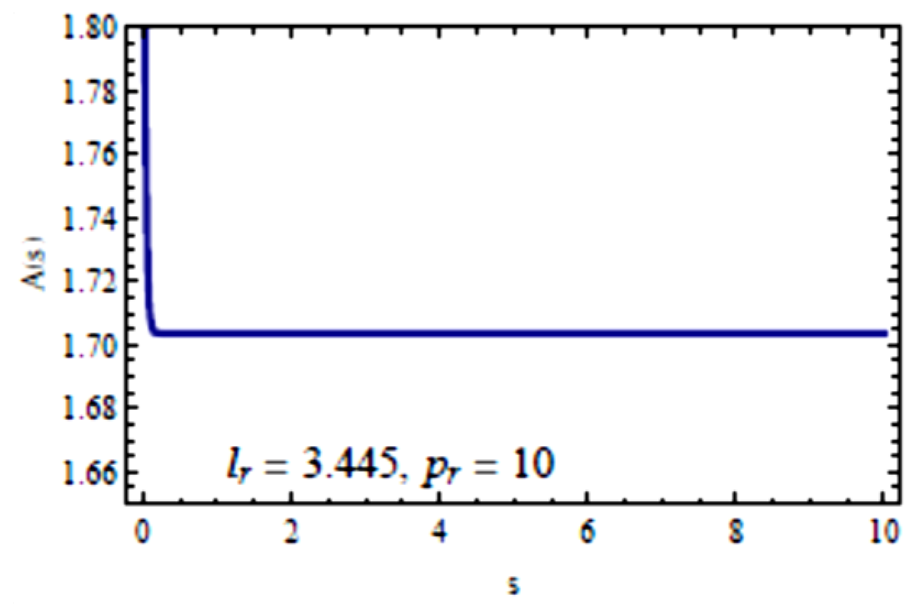

Fig.16. Phase portrait diagram for $A(s)$ and $s$, shows that $A(s)$ is stable when $s$ increases.

\section{Conclusions}

This paper deals with a combined effect of internal heating and gravity modulation on oscillatory convection. By performing a weak nonlinear stability analysis using the Gingburg-Landau equation, following conclusions are drawn:

a) The nature and the effect of viscoelastic fluid is in agreement with followed the studies of Kim et al. [20], Bhadauria and Kiran [26]-[29].

b) The effects of amplitude and frequency of modulation are, respectively to increase and decreases the heat transfer.

c) The effect of internal Rayleigh number is to increase the heat transport in the system.

d) The equilibrium point of the Ginzburg-Landau equation losses its stability as $\lambda_{I}$ increases.

e) If $p_{r}>0$ and $l_{r}>0$, then the amplitude equation gives two more equilibrium points, which are stable while the origin losses its stability.

\section{Acknowledgment}

The authors are grateful to the anonymous referees for the time, effort, and extensive comments which improve the quality of the paper. M.K. Singh and A. Singh gratefully acknowledge the financial assistance from Babasaheb Bhimrao Ambedkar University, Lucknow, India, in the form of a research fellowship.

\section{Nomenclature}

$$
\begin{gathered}
A(s) \text { - amplitude of convection } \\
a \text { - wave number }
\end{gathered}
$$




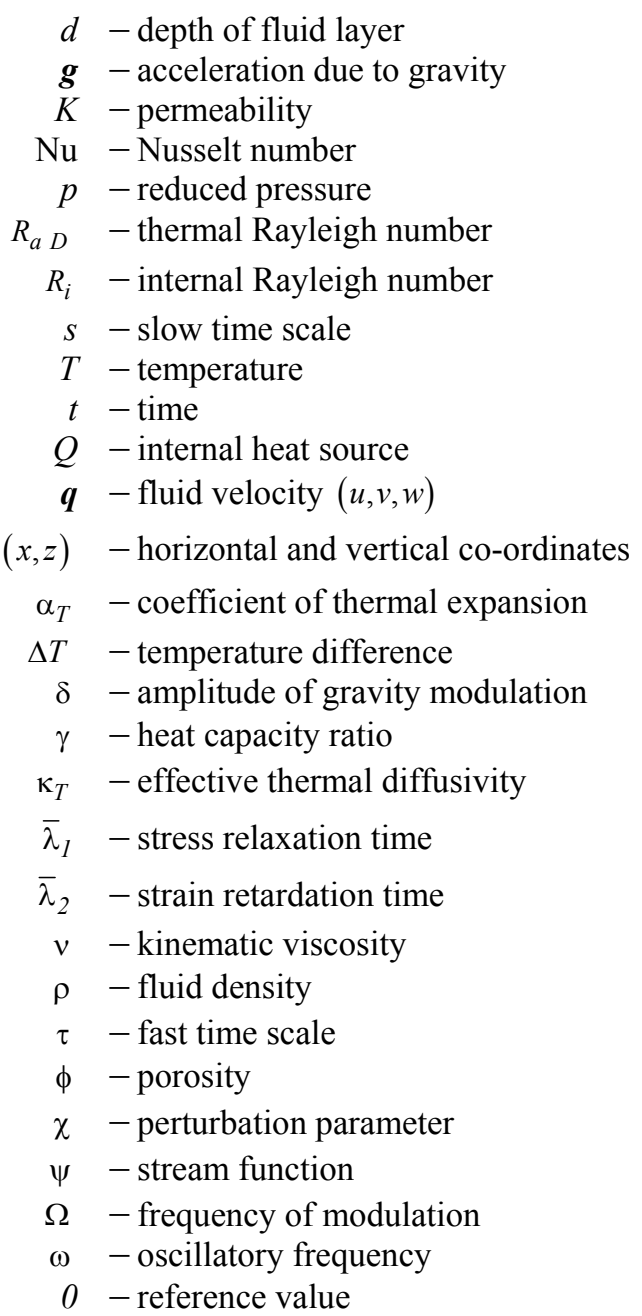

\section{Superscripts}

, perturbed quantity

* - dimensionless quantity

\section{Subscripts}

$$
\begin{aligned}
& b \text { basic state } \\
& c \text { critical } \\
& \nabla^{2}=\frac{\partial^{2}}{\partial x^{2}}+\frac{\partial^{2}}{\partial y^{2}}+\frac{\partial^{2}}{\partial z^{2}}
\end{aligned}
$$

\section{References}

[1] Ingham D.B. and Pop I.eds. (2005): Transport Phenomena in Porous Media. - Vol.3, 1st Edn. Oxford: Elsevier.

[2] Nield D.A. and Bejan A. (2013): Convection in Porous Media. - 3rd edn. New York: Springer:

[3] Vafai K.ed. (2000): Handbook of Porous Media. - New York: Marcel Dekker.

[4] Gresho P.M. and Sani R. (1970): The effects of gravity modulation on the stability of a heated fluid layer. - J. Fluid Mech., vol.40, pp.783-806. 
[5] Bhadauria B.S. and Bhatia P.K. Lokenath D. (2005): Convection in Hele-Shaw cell with parametric excitation. Int. J. Non-Linear Mech., vol.40, No.4, pp.475-484.

[6] Malashetty M.S. and Padmavathi V (1997): Effect of gravity modulation on the onset of convection in a fluid and porous layer. - Int. J. Engg. Sci., vol.35, pp.829-839.

[7] Das R. and Pop I. (2000): The effect of G-jitter on vertical free convection boundary-layer flow in porous media. -Int Comm Heat Mass Transf, vol.27, No.3, pp.415-424.

[8] Govender S. (2005a): Weak non-linear analysis of convection in a gravity modulated porous layer. - Transp. Porous Med., vol.60, pp.33-42.

[9] Siddhavaram V.K. and Homsy G.M. (2006): The effects of gravity modulation on fluid mixing Part 1. Harmonic modulation. - J. Fluid Mech., vol.562, pp.445-475.

[10] Saravanan S. and Sivakumar T. (2011): Thermovibrational instability in a fluid saturated anisotropic porous medium. - ASME J. Heat Transf., 133:051601.1-051601.9.

[11] Malashetty M.S. and Swamy M. (2011): Effect of gravity modulation on the onset of thermal convection in rotating fluid and porous layer. - Phys. Fluids, vol.23, No.6, 064108.

[12] Bhadauria B.S., Srivastava A. K., Sacheti N.C. and Chandran P. (2012): Gravity modulation of thermal instability in a viscoelastic fluid-saturated-anisotropic porous medium. - Z. Naturforch, vol.67a, pp.1-9.

[13] Bhadauria B.S., Siddheshwar P.G., Kumar J. and Suthar O. P. (2012): Non-linear stability analysis of temperature / gravity modulated Rayleigh-Benard convection in a porous medium. - Transp. Porous Med., vol.92, pp.633647.

[14] Siddheshwar P.G., Bhadauria B.S., Mishra P. and Srivastava A.K. (2012): Study of heat transport by stationary magneto-convection in a Newtonian liquid under temperature or gravity modulation using Ginzburg-Landau model. - Int. J. Nonlinear Mech., vol.47, pp.418-425.

[15] Siddheshwar P.G., Bhadauria B.S. and Srivastava A. (2012): An analytical study of nonlinear double diffusive convection in a porous medium with temperature modulation / gravity modulation. - Transp. Porous Med., vol.91, pp.585-604.

[16] Bhadauria B.S., Siddheshwar P.G. and Suthar O. P. (2012): Non-linear thermal instability in a rotating viscous fluid layer under temperature/gravity modulation. - ASME J. Heat Trans., vol.134, No.10,102502, doi.10.1115/1.4006868.

[17] Green T. III. (1968): Oscillating convection in an elasticoviscous liquid. - Phys. Fluids, vol.11, 1410. doi.10.1063/1.1692123

[18] Vest C.M. and Arpaci V.S. (1969): Overstability of a viscoelastic fluid layer heated from below. - J. Fluid Mech., vol.36, pp.13-623.

[19] Bhatia P.K. and Steiner J.M. (1972): Convective instability in a rotating viscoelastic fluid layer. - ZAMM, vol.52, pp.321-327.

[20] Kim M.C., Lee S.B., Kim S. and Chung B.J. (2003): Thermal instability of viscoelastic fluids in porous media. Int. J. Heat Mass Transfer, vol.46, pp.5065-5072.

[21] Malashetty M.S. and Kulkarni S. (2009): The convective instability of Maxwell fluid-saturated porous layer using a thermal non-equilibrium model. - J. Non-Newton Fluid Mech., vol.162, No.3, pp.29-37.

[22] Wang S. and Tan W. (2011): Stability analysis of Soret-driven double-diffusive convection of Maxwell fluid in a porous medium. - Int. J. Heat Fluid Flow, vol.32, No.1, pp.88-94.

[23] Kumar A. and Bhadauria B.S. (2011): Non-linear two dimensional double diffusive convection in a rotating porous layer saturated by a viscoelastic fluid. - Transp. Porous Med, vol.87, pp.229-250

[24] Kumar A. and Bhadauria B.S. (2011): Double diffusive convection in a porous layer saturated with viscoelastic fluid using a thermal non-equilibrium model. - Phys. Fluids, vol.223, pp. 967-983.

[25] Kumar A. and Bhadauria B.S. (2011): Thermal instability in a rotating anisotropic porous medium saturated with viscoelastic fluid. - Int. J. Nonlinear Mech., vol.46, pp.47-56. 
[26] Bhadauria B.S. and Kiran P. (2014): Weak non-linear oscillatory convection in a viscoelastic fluid saturated porous medium under gravity modulation. - Transp. Porous Med., vol.104, pp.451-467.

[27] Bhadauria B.S. and Kiran P. (2014): Weak non-linear oscillatory convection in a viscoelastic fluid layer under gravity modulation. - Int. J. Nonlinear Mech., vol.65, pp.133-140.

[28] Bhadauria B.S. and Kiran P. (2014a): Weakly non-linear oscillatory convection in a viscoelastic fluid saturating porous medium under temperature modulation. - Int. J. Heat Mass Transfer, vol.77, pp.843-851.

[29] Bhadauria B.S. and Kiran P. (2015): Chaotic and oscillatory magneto-convection in a binary viscoelastic fluid under G-jitter. - Int. J. Heat Mass Transfer, vol.84, pp.610-614.

[30] Haajizadeh M., Ozguc A.F. and Tien C.L. (1984): Natural convection in a vertical porous enclosure with internal heat generation. - Int. J. Heat Mass Transfer, vol.27, pp.1893-1902.

[31] Bhattacharya S.P. and Jena S.K. (1984): Thermal instability of a horizontal layer of micropolar fluid with heat source. - Proc. Indian Acad. Sci. (Math Sci), vol.93, No.1, pp.13-26.

[32] Parthiban C. and Patil P.R. (1997): Thermal instability in an anisotropic porous medium with internal heat source and inclined temperature gradient. - Int. Comm. Heat Mass Transfer, vol.24, No.7, pp.104-1058.

[33] Magyari E., Pop I. and Postelnicu, A. (2007): Effect of the source term on steady free convection boundary layer flows over a vertical plate in a porous medium Part I. - Transp. Porous Med., vol.67, pp.49-67.

[34] Bhadauria B.S., Kumar A., Kumar J., Sacheti N.C. and Chandran P. (2011): Natural convection in a rotating anisotropic porous layer with internal heat generation. - Transp. Porous Med., vol.90, No.2, pp.687-705.

[35] Bhadauria B.S. (2012): Double diffusive convection in a saturated anisotropic porous layer with internal heat source. - Transp. Porous Med., vol.92, pp.299-320.

[36] Bhadauria B.S., Hashim I. and Siddheshwar P.G. (2013): Effect of internal-heating on weakly non-linear stability analysis of Rayleigh-Benard convection under G-jitter. - Int. J. Nonlinear Mech., vol.54, pp.35-42.

[37] Bhadauria B.S, Hashim I. and Siddheshwar P.G. (2013): Study of heat transport in a porous medium under G-jitter and internal heating effects. - Transp. Porous Med., vol.96, pp.21-37.

[38] Bhadauria B.S., Hashim I. and Siddheshwar P.G. (2013): Effects of time-periodic thermal boundary conditions and internal heating on heat transport in a porous medium. - Transp. Porous Med., vol.97, pp.185-200.

[39] Srivastava A., Bhadauria B.S., Siddheshwar P.G. and Hashim I. (2013): Heat transport in an anisotropic porous medium saturated with variable viscosity liquid under g-jitter and internal heating effects. - Transp. Porous Med., vol.99, pp.359-376.

[40] Altawallbeh A.A., Bhadauria B.S. and Hashim I. (2013): Linear and nonlinear double-diffusive convection in a saturated anisotropic porous layer with Soret effect and internal heat source. - Int. J. Heat Mass Transf., vol.59, pp.103-111.

[41] Horton C.W. and Rogers F.T. (1945): Convection currents in a porous medium. - J. Appl. Phys., vol.16, pp.367370.

[42] Strogatz S.H. (2007): Non-Linear Dynamics and Chaos. - Levant Books, Edition-I Kolkata India .

[43] Kuznetsov Y A. (1998): Topological Equivalence, Bifurcations and Structural Stability of Dynamical Systems. In Elements of Applied Bifurcation Theory. Edition-II, Springer.

[44] Malkus W.V.R. and Veronis G. (1958): Finite amplitude cellular convection. - J. Fluid Mech., vol.4, pp.225-260.

[45] Venezian G. (1969) Effect of modulation on the onset of thermal convection. - J. Fluid Mech., vol.35, pp.243-254. 